\title{
HIPEC: The Complexities of Clinical Trials
}

\author{
David L. Bartlett, $\mathrm{MD}^{1,2}$ \\ ${ }^{1}$ Division of Surgical Oncology, University of Pittsburgh, Pittsburgh, Pennsylvania, USA \\ ${ }^{2}$ David C. Koch Regional Perfusion Center, University of Pittsburgh Cancer Institute, Pittsburgh, Pennsylvania, USA
}

The treatment of peritoneal surface malignancies presents unique and challenging problems to the cancer clinician. These patients more than many other cancer patients often suffer from significant and disabling symptoms as a direct result of local tumor progression. As a result, development in this field has largely focused on the disease site rather than histology. Innovative therapies have been largely driven by surgical oncologists who are often confronted with the challenge of controlling or palliating the locoregional progression in the absence of systemic disease. Traditionally, recurring problems were inevitable, and success was transient at best. More recent approaches have attempted to combine surgical debulking with locoregional administration of chemotherapy in an attempt to halt unrelenting locoregional progression of the disease. Offering innovative therapy to patients with no standard treatments available is part of what fulfills our mission as surgical oncologists.

Unfortunately, the field of peritoneal surface malignancies (HIPEC in particular) has a reputation for being based more on common sense than on experimental data. The anecdotal data and singleseries reports demonstrate promise, and small randomized trials have demonstrated remarkable survival advantages for HIPEC compared with standard therapy. Reported results for surgically treated peritoneal surface malignancies combined with HIPEC are similar to that obtained for other surgically treated cancers, such as liver resection for metastatic disease and pancreatic resection for cancer. However, the treatment is expensive financially and costly in terms of morbidity and potential effects

Published online March 7, 2008.

Address correspondence and reprint requests to: David L. Bartlett, MD; E-mail: bartlettdl@upmc.edu

Published by Springer Science+Business Media, LLC $\odot 2008$ The Society of Surgical Oncology, Inc. on patients' quality of life. Improved systemic chemotherapy options create a moving target in terms of expectations for survival, which makes interpretation of retrospective series difficult. The field is growing in numbers of practitioners at a considerable pace, but lagging behind in clinical trials to support the strategy. The easy answer is to run well-controlled phase I, phase II, and phase III clinical trials. Is this possible? The study by Harrison et al. ${ }^{1}$ is an excellent example of the kind of studies that are needed in the field of peritoneal surface malignancies and regional therapy, and the authors are to be commended for the enormous effort involved in performing a surgical trial with HIPEC. As with many well-constructed experiments and clinical trials, the results reported in this issue leave many questions unanswered and highlight many of the complexities of performing surgical trials for peritoneal malignancies.

The manuscript reports a phase I study of an innovative therapy for patients with peritoneal surface malignancies. The study attempted to define the toxicity and feasibility of intraoperative delivery of liposomal doxorubicin combined with hyperthermia in a recirculating perfusion of the abdominal cavity (a procedure known as HIPEC: hyperthermic intraperitoneal chemotherapy). In this trial, 21 patients were enrolled and treated with doses ranging from 15 to $100 \mathrm{mg} / \mathrm{m}^{2}$, with $100 \mathrm{mg} / \mathrm{m}^{2}$ being considered a safe dose for phase II studies. While no side effects directly related to the chemotherapy were encountered, other side effects related to the surgical procedure were experienced. Pain, discomfort, weight loss, energy loss, and decreased performance status accompanies major abdominal surgery and need to be considered as side effects of the whole treatment. Unfortunately, this trial failed to reach traditional phase I end points. An anastomotic leak was encountered at $100 \mathrm{mg} / \mathrm{m}^{2}$, which might be related to 
the drug impeding healing at that dose, or it might be caused by a technical mishap, or poor healing related to malnutrition, carcinomatosis, or hyperthermia. Nevertheless, the consequence of an anastamotic leak is quite significant, and it would be very difficult to continue to dose escalate if there was any chance that the leak was related to the dose of adriamycin. We have seen similar complications (or toxicities) in phase I studies that are difficult to define as drug related (unpublished). In a phase I study of early postoperative intraperitoneal 5-FU after HIPEC, we defined the dose-limiting toxicity of 5-FU as pancreatitis, which may well have been a complication from the debulking or hyperthermia. With the possibility that it could be related, the dose escalation was terminated in that study. The maximum tolerated dose of a drug is therefore very difficult to define in the context of HIPEC. Only if the toxicity encountered is expected based on the systemic profile of the drug can the dose be considered directly related to the toxicity. ${ }^{2}$ This is often not the case with HIPEC, yet dose escalation must be terminated because of unrelated complications making accurate determination of the maximum tolerated dose difficult.

Even when safe and tolerable doses for intraperitoneal chemotherapy can be defined, phase II studies are even more difficult. These are difficult to fund, and it is hard to accrue adequate numbers of appropriate, eligible patients. Once complete, consensus in interpretation of phase II trials in HIPEC is impossible. Because there is no measurable disease after surgical cytoreduction and HIPEC, there are no tumor response measurements. The only objective endpoints that can be validated are disease-free survival or overall survival. Survival results must be compared with the standard of care. Unfortunately, there are no good studies of modern systemic chemotherapy regimens in the treatment of isolated peritoneal carcinomatosis. There is the impression that these patients do worse than patients with liver or lung metastases, because abdominal pain and bowel obstruction become life-limiting at relatively small tumor burdens compared with what would cause liver or lung failure. However, patients with isolated peritoneal carcinomatosis are not examined in large databases such as the SEER database or most cancer registries. Even controlling for histology, many factors are presumed to be important in expected outcome such as the extent of disease before and after surgery, the disease-free interval prior to developing carcinomatosis, the tumor doubling time, and the biological aggressiveness of the cells. Comparing different HIPEC phase II studies can be dif- ficult because of variables in terms of drug type and dose, duration of treatment, level of hyperthermia, and extent of debulking. These variables combined with inadequate data on expected outcomes using standard treatments make phase II HIPEC studies difficult to perform and interpret.

Finally, phase III studies of HIPEC are even more difficult to perform and interpret. Single-institutional studies will not accrue adequate numbers of patients. Multi-institutional studies are difficult because of variability in surgeons' styles, perioperative care, and differing preconceived notions of how HIPEC should be delivered. It is difficult to accrue to randomized trials involving aggressive surgery, because patients often cannot comprehend that a "flip of the coin" could determine whether or not they undergo prolonged surgery with the associated risk of complications and death or perhaps go untreated for an imminently terminal condition. In addition, these trials are difficult to construct because the control treatment is a moving target that will inevitably place in doubt conclusions from randomized trials. Finally the cost per patient of a randomized trial could easily be between $\$ 50,000$ and $\$ 100,000$ if the entire surgery, drug, and perioperative care is included. Drug companies in general do not fund trials that are designed to use less of their product in a regional therapy setting compared with systemic use. The cost is also too high for device companies, philanthropic funds, or granting agencies. Even if a randomized trial is performed and results are positive, it will not define which aspect of HIPEC is important. Is it the extent of debulking, the heat, or the drug that is working? ${ }^{3}$ Should individual trials be performed examining each component of the therapy, or should we test the package as a whole and then tease out the ineffective parts that have unacceptable side effects later.

Despite these difficulties the anecdotal data, retrospective reviews, prospective database reports, phase II studies, and small phase III studies are compelling. Verwaal et al. reported in the Journal of Clinical Oncology almost a doubling in median survival for patients with peritoneal carcinomatosis from colon cancer (22.3 vs 12.6 months) using HIPEC. ${ }^{4}$ For many, this compilation of data is enough to consider the use of HIPEC as standard therapy. ${ }^{5}$ For others, skepticism and intolerance of the high morbidity associated with HIPEC leads to the pursuit of other options. So, how do we continue to move the field forward? Realizing the perils discussed above, I believe that the answer is to keep trying to gather data. We should continue to perform and publish trials such as that reported by Harrison et al. ${ }^{1}$ We need to 
follow outcomes prospectively for those treated as standard of care. Those interested in this field should standardize treatment approaches and move forward with multi-institutional phase II and phase III studies. Surgical and medical oncologists need to combine strategies. HIPEC should be combined with standard systemic chemotherapy options to provide incremental improvements rather than compared as an alternative to systemic chemotherapy. I can only imagine the multitude of excuses and nihilism that must have confronted Dr. Bernard Fisher and others who were performing the early randomized trials that revolutionized our management of breast cancer. Many of the excuses at that time would be similar to that discussed above for HIPEC. Their success should give present and future surgical oncologist the courage to forge a new path guided by well-constructed clinical trials.

\section{REFERENCES}

1. Harrison LE, Bryan M, Pliner L, et al. Phase I trial of pegylated liposomal doxorubicin with hyperthermic intraperitoneal chemotherapy in patients undergoing cytoreduction for advanced intra-abdominal malignancy. Ann Surg Oncol 2007; current.

2. Bartlett DL, Buell JF, Libutti SK, et al. A phase I trial of continuous hyperthermic peritoneal perfusion with tumor necrosis factor and cisplatin in the treatment of peritoneal carcinomatosis. Cancer 1998; 83:1251-61.

3. Miner TJ, Shia J, Jaques DP, et al. Long-term survival following treatment of pseudomyxoma peritonei: an analysis of surgical therapy. Ann Surg 2005; 241:300-8.

4. Verwaal VJ, van Ruth S, de Bree E, et al. Randomized trial of cytoreduction and hyperthermic intraperitoneal chemotherapy versus systemic chemotherapy and palliative surgery in patients with peritoneal carcinomatosis of colorectal cancer. J Clin Oncol 2003; 21:3737-43.

5. Esquivel J, Sticca R, Sugarbaker P, et al. Cytoreductive surgery and hyperthermic intraperitoneal chemotherapy in the management of peritoneal surface malignancies of colonic origin: a consensus statement. Society of Surgical Oncology. Ann Surg Oncol 2007; 14:128-33. 